\title{
Weed Control in Corn (Zea mays L.) as Influenced by Preemergence Herbicides
}

\author{
Travis W. Janak ${ }^{1}$ and W. James Grichar ${ }^{2}$ \\ ${ }^{1}$ B-H Genetics, 5933 FM 1157, Ganado, TX 77962, USA \\ ${ }^{2}$ Texas A\&M AgriLife Research and Extension Center, 10345 State Highway 44, Corpus Christi, TX 78406, USA
}

Correspondence should be addressed to W. James Grichar; w-grichar@tamu.edu

Received 4 February 2016; Accepted 14 April 2016

Academic Editor: Maria Serrano

Copyright (c) 2016 T. W. Janak and W. J. Grichar. This is an open access article distributed under the Creative Commons Attribution License, which permits unrestricted use, distribution, and reproduction in any medium, provided the original work is properly cited.

Field studies were conducted in central and south-central Texas from 2013 through 2015 to evaluate crop tolerance and efficacy of various preemergence herbicides alone and in combination for weed control in field corn. Acetochlor and pendimethalin alone, $S$-metolachlor plus mesotrione, and the three-way combination of $S$-metolachlor plus atrazine plus mesotrione provided the most consistent control of annual grasses including browntop panicum (Panicum fasciculatum L.), Texas millet (Urochloa texana L.), barnyardgrass (Echinochloa crus-galli L.), and sprawling signalgrass (Brachiaria reptans L.). Palmer amaranth [Amaranthus palmeri (S.) Wats.] control was at least $90 \%$ with fluthiacet-methyl plus pyroxasulfone, atrazine plus either acetochlor, alachlor, dimethenamid-P, $S$-metolachlor, or $S$-metolachlor plus mesotrione, saflufenacil plus dimethenamid-P, and $S$-metolachlor plus mesotrione. Hophornbeam copperleaf (Acalypha ostryifolia L.) was difficult to control; however, acetochlor, saflufenacil or pyroxasulfone alone, saflufenacil plus dimethenamid-P, and S-metolachlor plus mesotrione provided at least $90 \%$ control. Acetochlor or saflufenacil alone, thiencarbazone-methyl plus isoxaflutole, dimethenamid-P plus atrazine, rimsulfuron plus mesotrione, and saflufenacil plus dimethenamid-P controlled common sunflower (Helianthus annuus L.) at least $90 \%$. Corn injury was minimal $(\leq 3 \%)$ with all herbicides. In general, corn grain yield was greatest with herbicide treatments containing more than one active ingredient compared with a single active ingredient.

\section{Introduction}

Corn (Zea mays L.) weed management programs in Texas have traditionally relied on preemergence (PRE) applications of a broadleaf and grass herbicide for residual season-long weed control. These PRE programs usually have included atrazine in combination for broad-spectrum weed control. Atrazine is used on greater than $60 \%$ of the US corn ha, with the dose decreasing over time with most doses of no more than $1.12 \mathrm{~kg} / \mathrm{ha}$, and some growers do not apply in excess of $0.84 \mathrm{~kg} / \mathrm{ha}$ [1].

However, over the past twenty years the use of glyphosateresistant corn has been rapidly adopted and used extensively in corn grown areas across the state [2]. In 2009, nearly 61 million ha of soybean [Glycine max (L.) Merr.], corn, and cotton (Gossypium hirsutum L.) in the US contained a modified 5-enolpyruvylshikimate-3-phosphate synthase (EPSPS) gene that confers resistance to glyphosate [3]. The popularity of glyphosate-resistant crops, reduction of traditional herbicide and cultivation practices, and intense management of weeds using glyphosate as the predominant control strategy have caused a shift in weed populations $[4,5]$ and created a selective advantage for glyphosate-resistant weeds. Glyphosate-resistant weeds, specifically Amaranthus species, have become an issue across Texas and the US corn-producing areas $[6,7]$.

Atrazine and 4-hydroxyphenylpyruvate dioxygenase (HPPD) inhibiting herbicides are commonly used for weed control in corn and are effective in controlling glyphosateresistant weeds, including A. palmeri [8-10]. Atrazine can be applied PRE or POST alone or in tank-mixtures with several herbicides [11]. The HPPD-inhibiting herbicides have become popular among corn growers because of their broad-spectrum weed control, flexible application timing, tank-mix compatibilities, and crop safety [11-13]. 
Amaranthus species are some of the most common weed species found in annual crop production throughout the US [14]. A. palmeri is not ranked as the number one weed or even as a principal weed in several major crops, but it is a common weed in many major crops around the world and in Texas [15]. Up till the 1990s its distribution in North America was the southern half of the US [16]; however, since then it has become established in every state with the exception of North Dakota and Minnesota [17]. In Texas, A. palmeri can be found in all areas of the state [18]. A. palmeri and A. rudis Sauer are the two Amaranthus species with confirmed resistance to glyphosate across Texas [19].

A. palmeri is a dioecious, summer-annual species that is native to the desert southwest region of the US [20, 21]. Despite its origin, $A$. palmeri is able to survive in many diverse environments because of its biological characteristics [7, 22]. It has a lengthy germination window and robust growth habit and is a prolific seed producer [22-25] and these characteristics make control of this weed difficult.

A. rudis is an obligate outcrossing annual broadleaf weed that is capable of long-distance pollen dispersal [26]. It germinates optimally between $20 / 25$ and $30 / 35^{\circ} \mathrm{C}$ [27], has an aggressive growth habit, may grow $1.6 \mathrm{~mm}$ per growing degree day [24], and is capable of producing greater than 250,000 seeds per plant [22]. These factors make it a strong competitor with most crop plants.

Herbicide resistance complicates weed management in corn and many other crops. Estimates are that more than 1.2 million ha of cropland in the US is now affected by glyphosate-resistant Amaranthus species [28]. Also, weeds resistant to photosystem II- (PSII-) inhibiting herbicides, including atrazine, and HPPD-inhibiting herbicides have been documented [28]. Resistance to PSII inhibitors has been documented in seven monocot and 17 dicot species in the corn producing regions of the US [29].

The concerns pertaining to the overuse of atrazine including detection in surface and groundwater, rotational crop injury, and the development of triazine-resistant weeds as well as the increase in acreage with glyphosate-resistant weeds have resulted in questions from growers about the renewed use of PRE herbicides for early-season and possibly season-long weed control in corn. Although many relatively new soil-applied herbicides have been on the market for several years there is little field based information in the scientific literature on the efficacy of weeds commonly found in the south and central Texas corn growing regions. Therefore, the objective of this research was to evaluate the effect of various PRE herbicides for crop tolerance and weed control efficacy in these corn-producing regions.

\section{Materials and Methods}

Field studies were conducted during the 2013 through 2015 growing season in central Texas near Taylor $\left(30.5326^{\circ} \mathrm{N}\right.$, $97.4548^{\circ} \mathrm{W}$ ) and in south-central Texas near Ganado $\left(29.0438^{\circ} \mathrm{N}, 96.4849^{\circ} \mathrm{W}\right)$. Study sites were located in different fields within the same general area of each year. Soils at the Taylor location were a Burleson clay (fine, montmorillonitic, thermic Udic Pellusterts) with less than $1 \%$ organic matter and $7.6 \mathrm{pH}$ while soils at the Ganado location were a Houston Black clay (fine, montmorillonitic, thermic Udic Pellusterts) with less than $1 \%$ organic matter and $7.4 \mathrm{pH}$. Studies were arranged in a randomized complete block design with three replicates. Plot dimensions were two or four corn rows wide spaced 76 to $97 \mathrm{~cm}$ apart and 6.3 or $7.9 \mathrm{~m}$ long (depending on location). The corn hybrids BH 8846RR (2013), BH 8844VTTP (2014), and BH 8475SS (2015) were planted mid- to late-February near Taylor and late-February to early-March near Ganado in each year to a depth of 2.5 to $3.5 \mathrm{~cm}$ at the rate of 54,000 to 65,500 seeds $/$ ha.

Herbicides were applied within 5 to $7 \mathrm{~d}$ after planting with a $\mathrm{CO}_{2}$-pressurized backpack sprayer with Teejet 11002 flat fan nozzles (Spraying Systems Co., North Avenue and Schmale Road, Wheaton, IL 60188) using a pressure of $180 \mathrm{kPa}$ and calibrated to deliver 140 or $187 \mathrm{~L} / \mathrm{ha}$ (depending on location). Herbicide treatments are shown in Table 1. An untreated check was included for comparison at each location. All herbicide doses were based on the US label dose with the exception of the acetochlor (74.8\% formulation) dose which was applied at $2 \mathrm{x}$ of the labeled rate throughout the study by mistake. Once the error was realized, it was decided to maintain this dose throughout the study.

Weed populations varied from year to year and were from naturally occurring populations. At the Taylor location, Panicum fasciculatum populations in 2013 were moderate (3 to 4 plants $/ \mathrm{m}^{2}$ ) while in 2014 populations were higher (6 to 8 plants $/ \mathrm{m}^{2}$ ). Echinochloa crus-galli populations in 2015 ranged from 4 to 8 plants $/ \mathrm{m}^{2}$. At Ganado, Urochloa texana populations ranged from 6 to 10 plants $/ \mathrm{m}^{2}$. Brachiaria reptans populations were moderate to heavy (5 to 10 plants $/ \mathrm{m}^{2}$ ). A. palmeri populations varied from 4 to 8 plants $/ \mathrm{m}^{2}$ at the Taylor location to 2 to 10 plants $/ \mathrm{m}^{2}$ at the Ganado location. Acalypha ostryifolia populations at Taylor in both years were low to moderate (2 to 6 plants $/ \mathrm{m}^{2}$ ) while Helianthus annuus populations ranged from 2 to 6 plants $/ \mathrm{m}^{2}$ depending on the year. Crop injury and weed control were visually estimated on a scale of 0 to 100 (0 indicating no control or injury and 100 indicating complete control or plant death). Crop injury consisted of plant stunting and early-season (30 d after herbicide application) and late-season (95 to $140 \mathrm{~d}$ after application) crop injury was taken. Late-season weed control ratings (95 to 140 days after herbicide application) are presented for all weeds with the exception of $A$. palmeri control at Ganado in 2015 where populations of this weed were low $\left(<4\right.$ plants $\left./ \mathrm{m}^{2}\right)$ and somewhat inconsistent. Crop yield was determined by hand harvesting $3.8 \mathrm{~m}$ of each plot, shelling the kernels from the corn ear, and then weighing the kernels. Crop weights were adjusted to $12 \%$ moisture.

Visual estimates of weed control and corn injury were arcsine square root transformed prior to analysis of variance. Data are presented in their original form since the transformation did not alter interpretation. Means were compared with Fisher's Protected LSD test at the 5\% probability level [30]. The nontreated check was not included in the weed control or corn injury analysis but was included in corn yield analysis. 
TABLE 1: Herbicides, composition, and manufacturer.

\begin{tabular}{|c|c|c|c|}
\hline Common name & Trade name & Composition (\%) & Manufacturer \\
\hline Atrazine $(\mathrm{A})$ & AAtrex & 42.0 & Syngenta Crop Protection \\
\hline S-Metolachlor (S) & Dual Magnum & 83.6 & Syngenta Crop Protection \\
\hline$(\mathrm{S})+(\mathrm{A})$ & Bicep II Magnum & $26.1+33.0$ & Syngenta Crop Protection \\
\hline Mesotrione (M) & Callisto & 40.0 & Syngenta Crop Protection \\
\hline$(\mathrm{A})+(\mathrm{S})+(\mathrm{M})+$ bicyclopyrone & Acuron & $10.93+23.4+2.6+0.65$ & Syngenta Crop Protection \\
\hline Fluthiacet-methyl $(\mathrm{F})+$ pyroxasulfone $(\mathrm{P})$ & Anthem & $0.69+22.61$ & FMC Corporation \\
\hline$(\mathrm{F})+(\mathrm{P})+(\mathrm{A})$ & Anthem ATZ & $0.15+5.15+42.5$ & FMC Corporation \\
\hline Isoxaflutole & Balance Flexx & 20.0 & Bayer CropScience \\
\hline Alachlor $+(A)$ & Bullet & $25.4+14.5$ & Monsanto Company \\
\hline Thiencarbazone-methyl + isoxaflutole & Corvus & $7.6+19.0$ & Bayer CropScience \\
\hline Acetochlor $+(\mathrm{A})$ & Degree Xtra & $29.0+14.5$ & Monsanto Company \\
\hline Dimethenamid-P + (A) & Guardsman Max & $18.2+35.3$ & BASF Corporation \\
\hline Acetochlor (74.8\% formulation) & Harness & 75.9 & Monsanto Company \\
\hline Rimsulfuron + (M) & Instigate & $4.17+41.67$ & DuPont Crop Protection \\
\hline Rimsulfuron + thifensulfuron-methyl & Leadoff & $16.7+16.7$ & DuPont Crop Protection \\
\hline$(\mathrm{S})+(\mathrm{A})+(\mathrm{M})$ & Lexar & $19.0+19.0+2.44$ & Syngenta Crop Protection \\
\hline Dimethenamid-P & Outlook & 63.9 & BASF Corporation \\
\hline Pendimethalin & Prowl $\mathrm{H}_{2} \mathrm{O}$ & 38.7 & BASF Corporation \\
\hline Saflufenacil & Sharpen & 29.74 & BASF Corporation \\
\hline Saflufenacil + dimethenamid-P & Verdict & $6.24+55.04$ & BASF Corporation \\
\hline Acetochlor (33\% formulation) & Warrant & 33.3 & Monsanto Company \\
\hline$(\mathrm{S})+(\mathrm{M})$ & Zemax & $36.8+3.68$ & Syngenta Crop Protection \\
\hline$(\mathrm{P})$ & Zidua & 85.0 & BASF Corporation \\
\hline
\end{tabular}

TABLE 2: Rainfall amounts at test locations for twenty-one days following application of PRE herbicides.

\begin{tabular}{lcccccc}
\hline & \multicolumn{2}{c}{2013} & \multicolumn{2}{c}{2014} & \multicolumn{2}{c}{2015} \\
Day & Taylor & Ganado & Taylor & Ganado & Taylor & Ganado \\
& & \multicolumn{2}{c}{ Mm } \\
\hline $1-7$ & 29.5 & 0 & 2.8 & 0 & 7.4 & 3.3 \\
$8-14$ & 6.6 & 0 & 0.5 & 18.6 & 65.6 & 78.2 \\
$15-21$ & 7.3 & 0 & 0 & 0 & 0 & 3.3 \\
\hline
\end{tabular}

\section{Results and Discussion}

Since not all treatments were included in each year of the study no attempt was made to combine results over years or locations. Also, rainfall amounts varied from site to site and year to year affecting herbicide response (Table 2). Rainfall during the $7 \mathrm{~d}$ after the PRE herbicide treatments occurred at all locations with the exception of Ganado in 2013 and 2014 when no rainfall occurred. Rainfall between 8 and $14 \mathrm{~d}$ after the PRE application varied from no rainfall at Ganado in 2013 to $78.2 \mathrm{~mm}$ at Ganado in 2015 (Table 2). Rainfall 15 to $21 \mathrm{~d}$ after the PRE application was low at Taylor in 2013 and Ganado in 2015 and there was no rainfall at the other sites.

With respect to the annual grasses, Panicum fasciculatum and Urochloa texana were present in 2013 and 2014 at the Taylor and Ganado sites while Echinochloa crus-galli and Brachiaria reptans were present only in 2015 at Taylor and Ganado. All broadleaf weeds were present at the Taylor and Ganado locations. A. palmeri was present in 2013 and 2015 while Acalypha ostryifolia and Helianthus annuus were present in 2013 and 2014.

\subsection{Annual Grass Control}

Brachiaria reptans (Sprawling Signalgrass). This weed was present at Ganado only in 2015. Atrazine alone provides only $40 \%$ control while the high rate of acetochlor (at the $74.8 \%$ formulation) provided perfect control (Table 3). SMetolachlor alone, fluthiacet-methyl plus pyroxasulfone, thiencarbazone-methyl plus isoxaflutole, dimethenamid-P plus atrazine, or $S$-metolachlor plus mesotrione controlled this weed 93 to $99 \%$ while dimethenamid-P alone, fluthiacetmethyl plus pyroxasulfone plus atrazine, $S$-metolachlor plus atrazine, or saflufenacil plus dimethenamid-P provided 82 to $87 \%$ control. Taylor-Lovell and Wax [31] and Johnson et al. [32] reported that isoxaflutole did not improve Setaria faberi control when compared with atrazine plus $S$-metolachlor.

Echinochloa crus-galli (Barnyardgrass). This weed was present at Taylor only in 2015. Atrazine alone controlled barnyardgrass $33 \%$ while acetochlor (74.8\% formulation) or pendimethalin alone, acetochlor plus atrazine, $S$-metolachlor plus mesotrione, or $S$-metolachlor plus atrazine plus mesotrione provided 90 to $97 \%$ control (Table 3). The dinitroaniline herbicides, such as pendimethalin, are registered for use in over forty crops [33]. These herbicides usually provide excellent control of annual grasses [34-36]. 
TABLE 3: Annual grass control in corn with PRE herbicides.

\begin{tabular}{|c|c|c|c|c|c|c|c|}
\hline \multirow{6}{*}{ Treatment } & \multirow{6}{*}{$\begin{array}{c}\text { Dose } \\
\text { Product/ha }\end{array}$} & \multicolumn{2}{|c|}{ PANFA $^{\mathrm{a}}$} & \multicolumn{2}{|c|}{ UROTE } & \multirow{2}{*}{$\begin{array}{c}\text { ECHCG } \\
2015\end{array}$} & \multirow{2}{*}{$\begin{array}{c}\text { BRARE } \\
2015\end{array}$} \\
\hline & & 2013 & 2014 & 2013 & 2014 & & \\
\hline & & \multicolumn{2}{|c|}{ Taylor } & Ganado & Ganado & Taylor & Ganado \\
\hline & & \multicolumn{6}{|c|}{ Days after treatment } \\
\hline & & 95 & 138 & 109 & 112 & 101 & 42 \\
\hline & & \multicolumn{6}{|c|}{$\%$} \\
\hline Atrazine (A) & $2.34 \mathrm{~L}$ & 33 & 3 & 23 & 0 & 33 & 40 \\
\hline Fluthiacet-methyl + pyroxasulfone $(\mathrm{P})$ & $0.75 \mathrm{~L}$ & - & - & - & - & 58 & 99 \\
\hline Fluthiacet-methyl + (P) + (A) & $2.62 \mathrm{~L}$ & - & - & - & - & 40 & 86 \\
\hline S-Metolachlor (S) & $1.59 \mathrm{~L}$ & 82 & 57 & 78 & 75 & 68 & 93 \\
\hline Isoxaflutole & $0.47 \mathrm{~L}$ & 80 & 38 & 94 & 80 & 67 & 65 \\
\hline$(\mathrm{S})+(\mathrm{A})$ & $4.96 \mathrm{~L}$ & 85 & 53 & 86 & 83 & 63 & 82 \\
\hline Alachlor + (A) & $8.23 \mathrm{~L}$ & 99 & - & 89 & - & - & - \\
\hline Mesotrione (M) & $0.47 \mathrm{~L}$ & 37 & 8 & 53 & 88 & 55 & 10 \\
\hline Thiencarbazone-methyl + isoxaflutole & $0.19 \mathrm{~L}$ & 47 & 15 & 98 & 72 & 73 & 93 \\
\hline Acetochlor $+(A)$ & $8.23 \mathrm{~L}$ & 72 & 0 & 98 & 86 & 90 & 72 \\
\hline$(\mathrm{S})+(\mathrm{A})+(\mathrm{M})+$ bicyclopyrone & $2.90 \mathrm{~L}$ & - & - & - & - & 65 & 65 \\
\hline Dimethenamid-P + (A) & $4.12 \mathrm{~L}$ & 55 & 45 & 85 & 58 & 89 & 97 \\
\hline Acetochlor $(74.8 \%)$ & $8.23 \mathrm{~L}$ & - & 83 & - & 73 & 97 & 100 \\
\hline Rimsulfuron + (M) & $0.47 \mathrm{~L}$ & 60 & 33 & 77 & 47 & - & - \\
\hline Rimsulfuron + thifensulfuron-methyl & $0.09 \mathrm{~L}$ & 74 & 10 & 60 & 61 & 40 & 47 \\
\hline$(\mathrm{S})+(\mathrm{A})+(\mathrm{M})$ & $7.02 \mathrm{~L}$ & 98 & 44 & 83 & 73 & 92 & 76 \\
\hline Dimethenamid-P & $1.22 \mathrm{~L}$ & 78 & 53 & 55 & 63 & 73 & 87 \\
\hline Pendimethalin & $3.55 \mathrm{~L}$ & 96 & 52 & 86 & 99 & 97 & 58 \\
\hline Saflufenacil & $0.19 \mathrm{~L}$ & 69 & 7 & 81 & 23 & 33 & 78 \\
\hline Saflufenacil + dimethenamid-P & $1.12 \mathrm{~L}$ & 61 & 28 & 92 & 78 & 57 & 83 \\
\hline Acetochlor (33\%) & $3.55 \mathrm{~L}$ & 75 & 10 & 78 & 96 & 63 & 49 \\
\hline$(\mathrm{S})+(\mathrm{M})$ & $7.02 \mathrm{~L}$ & 98 & 65 & 67 & 95 & 96 & 94 \\
\hline (P) & $210.16 \mathrm{G}$ & 88 & 37 & 75 & 99 & 42 & 78 \\
\hline Untreated & - & 0 & 0 & 0 & 0 & 0 & 0 \\
\hline LSD $(0.05)$ & & 33 & 33 & 22 & 48 & 29 & 33 \\
\hline
\end{tabular}

${ }^{a}$ Bayer code for weeds: BRARE: Brachiaria reptans L.; ECHCG, Echinochloa crus-galli L.; PANFA, Panicum fasciculatum L.; UROTE, Urochloa texana (Buckl.) R. Webster.

${ }^{\mathrm{b}} \mathrm{L}=$ liters; $\mathrm{G}=$ grams.

Panicum fasciculatum (Browntop Panicum). In 2013, pendimethalin alone, alachlor plus atrazine, $S$-metolachlor plus mesotrione, or $S$-metolachlor plus atrazine plus mesotrione provided $96 \%$ or better browntop panicum control while isoxaflutole, $S$-metolachlor, and pyroxasulfone alone and $S$-metolachlor plus atrazine controlled this weed 80 to $88 \%$ (Table 3 ). In 2014, only acetochlor (74.8\% formulation) provided acceptable control (83\%). The lack of effective control in 2014 can be attributed to higher plant populations at the test site in 2014 compared to 2013 and also the low rainfall amounts after PRE application in 2014 (Table 2). Since many of the PRE herbicides can volatilize and photodegrade on the soil surface over time, these herbicides need to be mechanically incorporated or need rainfall or irrigation to move these herbicides into the weed seed zone [37-39] which explains the erratic control noted with these herbicides under the drought conditions observed at Taylor in 2014.
Urochloa texana (Texas Millet). In 2013, atrazine alone controlled only $23 \%$ while isoxaflutole alone, thiencarbazonemethyl plus isoxaflutole, acetochlor plus atrazine, or saflufenacil plus dimethenamid-P controlled $U$. texana at least $92 \%$ (Table 3). Pendimethalin or saflufenacil alone, atrazine plus either S-metolachlor, alachlor, or dimethenamid-P, and the three-way combination of $S$-metolachlor plus atrazine plus mesotrione provided 81 to $89 \%$ control. In 2014, acetochlor, pendimethalin, or pyroxasulfone alone or $S$-metolachlor plus mesotrione controlled this weed at least 95\% while isoxaflutole or mesotrione alone and atrazine plus either acetochlor or $S$-metolachlor provided 80 to $89 \%$ control (Table 3 ).

$S$-Metolachlor alone provided 75 to $78 \%$ U. texana control compared with 75 to $99 \%$ control with pyroxasulfone. Typically, $S$-metolachlor alone provides poor control of this weed $[40,41]$. With high populations of $U$. texana, Grichar et al. [40] reported less than $70 \%$ control with 1.7 and $3.4 \mathrm{~kg} / \mathrm{ha}$ 
TABLE 4: Amaranthus palmeri control in corn with PRE herbicides.

\begin{tabular}{|c|c|c|c|c|c|}
\hline \multirow{5}{*}{ Treatment } & \multirow{5}{*}{$\begin{array}{c}\text { Dose } \\
\text { Product/ha }\end{array}$} & \multicolumn{2}{|c|}{2013} & \multicolumn{2}{|c|}{2015} \\
\hline & & Taylor & Ganado & Taylor & Ganado \\
\hline & & \multicolumn{4}{|c|}{ Days after treatment } \\
\hline & & 95 & 109 & 101 & 44 \\
\hline & & \multicolumn{4}{|c|}{$\%$} \\
\hline Atrazine (A) & $2.34 \mathrm{~L}$ & 73 & 99 & 79 & 72 \\
\hline Fluthiacet-methyl $(\mathrm{F})+$ pyroxasulfone $(\mathrm{P})$ & $0.75 \mathrm{~L}$ & - & - & 99 & 98 \\
\hline$(\mathrm{F})+(\mathrm{P})+(\mathrm{A})$ & $2.62 \mathrm{~L}$ & - & - & 40 & 93 \\
\hline Isoxaflutole & $0.47 \mathrm{~L}$ & 100 & 98 & 51 & 67 \\
\hline S-Metolachlor (S) & $1.59 \mathrm{~L}$ & 100 & 76 & 99 & 69 \\
\hline$(\mathrm{S})+(\mathrm{A})$ & $4.96 \mathrm{~L}$ & 100 & 99 & 99 & 92 \\
\hline Alachlor $+(\mathrm{A})$ & $8.23 \mathrm{~L}$ & 100 & 99 & - & - \\
\hline Mesotrione $(\mathrm{M})$ & $0.47 \mathrm{~L}$ & 99 & 61 & 71 & 52 \\
\hline Thiencarbazone-methyl + isoxaflutole & $0.19 \mathrm{~L}$ & 100 & 92 & 99 & 83 \\
\hline$(\mathrm{S})+(\mathrm{A})+(\mathrm{M})+$ bicyclopyrone & $2.90 \mathrm{~L}$ & - & - & 99 & 72 \\
\hline Acetochlor $+(\mathrm{A})$ & $8.23 \mathrm{~L}$ & 100 & 100 & 99 & 93 \\
\hline Dimethenamid-P + (A) & $4.12 \mathrm{~L}$ & 100 & 100 & 99 & 95 \\
\hline Acetochlor $(74.8 \%)$ & $8.23 \mathrm{~L}$ & - & - & 100 & 100 \\
\hline Rimsulfuron $+(\mathrm{M})$ & $0.47 \mathrm{~L}$ & 100 & 27 & - & - \\
\hline Rimsulfuron + thifensulfuron-methyl & $0.09 \mathrm{~L}$ & 99 & 90 & 98 & 37 \\
\hline$(\mathrm{S})+(\mathrm{A})+(\mathrm{M})$ & $7.02 \mathrm{~L}$ & 100 & 97 & 99 & 90 \\
\hline Dimethenamid-P & $1.22 \mathrm{~L}$ & 98 & 53 & 96 & 92 \\
\hline Pendimethalin & $3.55 \mathrm{~L}$ & 97 & 83 & 98 & 47 \\
\hline Saflufenacil & $0.19 \mathrm{~L}$ & 99 & 72 & 70 & 73 \\
\hline Saflufenacil + dimethenamid-P & $1.12 \mathrm{~L}$ & 100 & 95 & 99 & 100 \\
\hline Acetochlor (33\%) & $3.55 \mathrm{~L}$ & 100 & 50 & 99 & 88 \\
\hline$(\mathrm{S})+(\mathrm{M})$ & $7.02 \mathrm{~L}$ & 100 & 91 & 100 & 94 \\
\hline (P) & $210.16 \mathrm{G}$ & 100 & 91 & 99 & 84 \\
\hline Untreated & - & 0 & 0 & 0 & 0 \\
\hline $\operatorname{LSD}(0.05)$ & & 17 & 27 & 22 & 24 \\
\hline
\end{tabular}

${ }^{\mathrm{a}} \mathrm{L}=$ liters; $\mathrm{G}=$ grams.

of metolachlor in dryland peanut (Arachis hypogaea L.) and 25 to $76 \%$ control under irrigated conditions. Steele et al. [41] reported that pyroxasulfone, at a 10-fold lower use rate than $S$-metolachlor, controlled $U$. texana 84 to $96 \%$ while $S$ metolachlor provided 75 to $85 \%$ control when rated 9 weeks after treatment. They attributed the results to the longer residual activity of pyroxasulfone [42].

\subsection{Broadleaf Weed Control}

Amaranthus palmeri (Palmer Amaranth). At Taylor in 2013, under moderate weed pressure ( 4 plants $\left./ \mathrm{m}^{2}\right)$, all herbicides, with the exception of atrazine (73\%), provided at least $97 \%$ control while in 2015 under higher populations ( 8 plants $/ \mathrm{m}^{2}$ ), atrazine controlled $79 \%$ while isoxaflutole, mesotrione, or saflufenacil controlled this weed no better than 71\% (Table 4). All other herbicide treatments provided at least $96 \%$ control.

At the Ganado location, in 2013 and 2015, control was more erratic than at the Taylor location. This may be due to the higher weed populations noted in 2013 (10 plants $\left./ \mathrm{m}^{2}\right)$ and variable populations in 2015. In 2013, either atrazine or isoxaflutole alone, acetochlor, alachlor, $S$-metolachlor, or dimethenamid plus atrazine or the three-way combination of $S$-metolachlor plus atrazine plus mesotrione provided 97 to $100 \%$ A. palmeri control while mesotrione, dimethenamid-P, or acetochlor (33\%) alone and rimsulfuron plus mesotrione controlled this weed $61 \%$ or less (Table 4 ). In 2015, acetochlor (74.8\%) alone, dimethenamid-P plus atrazine, fluthiacetmethyl plus pyroxasulfone, and saflufenacil plus dimethenamid-P controlled A. palmeri at least 95\% while isoxaflutole, mesotrione, $S$-metolachlor, and pendimethalin alone and rimsulfuron plus thifensulfuron-methyl controlled less than $70 \%$.

In previous research, mesotrione applied PRE controlled Amaranthus hybridus L. but control of Ipomoea spp. and Chenopodium album $\mathrm{L}$. was inconsistent and dependent upon a timely rainfall following application [43-45]. Armel et al. [43] reported improved weed control with mixtures of 
TABLE 5: Acalypha ostryifolia and Helianthus annuus control in corn with PRE herbicides.

\begin{tabular}{|c|c|c|c|c|c|}
\hline \multirow{5}{*}{ Treatment } & \multirow{5}{*}{$\begin{array}{c}\text { Dose } \\
\text { Product/ha }{ }^{b}\end{array}$} & \multicolumn{2}{|c|}{$\mathrm{ACCOS}^{\mathrm{a}}$} & \multicolumn{2}{|c|}{ HELAN } \\
\hline & & 2013 & 2014 & 2013 & 2014 \\
\hline & & \multicolumn{4}{|c|}{ Days after treatment } \\
\hline & & 95 & 109 & 95 & 48 \\
\hline & & \multicolumn{4}{|c|}{$\%$} \\
\hline Atrazine & $2.34 \mathrm{~L}$ & 38 & 80 & 73 & 77 \\
\hline Isoxaflutole & $0.47 \mathrm{~L}$ & 77 & 98 & 100 & 79 \\
\hline$S$-Metolachlor + atrazine & $4.96 \mathrm{~L}$ & 76 & 90 & 100 & 85 \\
\hline Alachlor + atrazine & $8.23 \mathrm{~L}$ & 93 & - & 98 & - \\
\hline Mesotrione & $0.47 \mathrm{~L}$ & 55 & 60 & 99 & 60 \\
\hline Thiencarbazone-methyl + isoxaflutole & $0.19 \mathrm{~L}$ & 100 & 77 & 98 & 100 \\
\hline Acetochlor + atrazine & $8.23 \mathrm{~L}$ & 79 & - & 99 & - \\
\hline S-Metolachlor & $1.59 \mathrm{~L}$ & 79 & 83 & 97 & 77 \\
\hline Dimethenamid-P + atrazine & $4.12 \mathrm{~L}$ & 80 & 72 & 97 & 93 \\
\hline Acetochlor ( $74.8 \%$ formulation) & $8.23 \mathrm{~L}$ & - & 99 & - & 97 \\
\hline Rimsulfuron + mesotrione & $0.47 \mathrm{~L}$ & 60 & 67 & 97 & 93 \\
\hline Rimsulfuron + thifensulfuron-methyl & $0.09 \mathrm{~L}$ & 98 & 70 & 87 & 99 \\
\hline$S$-Metolachlor + atrazine + mesotrione & $7.02 \mathrm{~L}$ & 98 & 74 & 100 & 87 \\
\hline Dimethenamid-P & $1.22 \mathrm{~L}$ & 72 & 82 & 97 & 79 \\
\hline Pendimethalin & $3.55 \mathrm{~L}$ & 69 & 85 & 95 & 58 \\
\hline Saflufenacil & $0.19 \mathrm{~L}$ & 96 & 98 & 100 & 90 \\
\hline Saflufenacil + dimethenamid-P & $1.12 \mathrm{~L}$ & 99 & 98 & 100 & 97 \\
\hline Acetochlor (33\% formulation) & $3.55 \mathrm{~L}$ & 92 & 63 & 100 & 72 \\
\hline$S$-Metolachlor + mesotrione & $7.02 \mathrm{~L}$ & 92 & 93 & 99 & 98 \\
\hline Pyroxasulfone & $210.16 \mathrm{G}$ & 96 & 93 & 97 & 55 \\
\hline Untreated & - & 0 & 0 & 0 & 0 \\
\hline LSD (0.05) & & 34 & 30 & 20 & 36 \\
\hline
\end{tabular}

a Bayer code for weeds: ACCOS: Acalypha ostryifolia Riddell; HELAN: Helianthus annuus L.

${ }^{\mathrm{b}} \mathrm{L}=$ liters; $\mathrm{G}=$ grams.

mesotrione plus acetochlor or atrazine over that of mesotrione alone. As seen in this study, the combination of mesotrione with metolachlor plus atrazine has enhanced weed control in other studies [43].

Acalypha ostryifolia (Hophornbeam Copperleaf). In 2013, thiencarbazone-methyl plus isoxaflutole provided perfect control (100\%) while acetochlor (33\% formulation), saflufenacil or pyroxasulfone alone, alachlor plus atrazine, rimsulfuron plus thifensulfuron-methyl, S-metolachlor plus atrazine plus mesotrione, saflufenacil plus dimethenamid$\mathrm{P}$, and $S$-metolachlor plus mesotrione controlled this weed at least 92\% (Table 5). Atrazine and mesotrione alone and rimsulfuron plus mesotrione provided unacceptable control $(\leq 60 \%)$.

In 2014, either acetochlor (74.8\% formulation), isoxaflutole, saflufenacil, or pyroxasulfone alone controlled A. ostryifolia at least $93 \%$ (Table 5). The combinations of $S$-metolachlor plus either atrazine or mesotrione and saflufenacil plus dimethenamid-P controlled this weed 90 to $98 \%$ while rimsulfuron plus either mesotrione or thifensulfuron-methyl and acetochlor (33\% formulation) provided 63 to $70 \%$ control.
Helianthus annuus (Common Sunflower). In 2013 under low weed pressure ( 2 to 3 plants $/ \mathrm{m}^{2}$ ), all herbicides with the exception of atrazine alone $(73 \%)$ and rimsulfuron plus thifensulfuron-methyl (87\%) controlled this weed at least 95\% (Table 5). In 2014, under slightly higher weed populations ( 4 to 6 plants $/ \mathrm{m}^{2}$ ) control was more variable. Acetochlor (74.8\% formulation) alone, thiencarbazone-methyl plus isoxaflutole, rimsulfuron plus thifensulfuron-methyl, saflufenacil plus dimethenamid-P, and $S$-metolachlor plus mesotrione controlled this weed at least $97 \%$. Mesotrione, pendimethalin, or pyroxasulfone alone provided unacceptable control $(\leq 60 \%)$.

The development of ALS-resistant $H$. annuus has limited the options for growers having to control this weed with POST herbicides $[46,47]$. Results from this study are consistent with previous findings that $H$. annuus control with herbicide systems containing isoxaflutole was at least $85 \%[32,47]$.

3.3. Corn Injury and Yield. Grain yields were obtained only in 2013 at both locations and 2015 at Taylor due to late-season time constraints. 
TABLE 6: Corn yield as influenced by herbicides.

\begin{tabular}{|c|c|c|c|c|}
\hline \multirow[b]{2}{*}{ Treatment } & \multirow[b]{2}{*}{$\begin{array}{c}\text { Dose } \\
\text { Product/ha }\end{array}$} & \multicolumn{2}{|c|}{2013} & \multirow{2}{*}{$\begin{array}{l}2015 \\
\text { Taylor }\end{array}$} \\
\hline & & Taylor & $\begin{array}{c}\text { Ganado } \\
\mathrm{kg} / \mathrm{ha}\end{array}$ & \\
\hline Fluthiacet-methyl $(\mathrm{F})+$ pyroxasulfone $(\mathrm{P})$ & $0.75 \mathrm{~L}$ & - & - & 9342 \\
\hline$(\mathrm{F})+(\mathrm{P})+$ atrazine & $2.62 \mathrm{~L}$ & - & - & 8092 \\
\hline Atrazine & $2.34 \mathrm{~L}$ & 5586 & 7695 & 7556 \\
\hline Isoxaflutole & $0.47 \mathrm{~L}$ & 5434 & 6980 & 7669 \\
\hline S-Metolachlor + atrazine & $4.96 \mathrm{~L}$ & 5396 & 7627 & 8582 \\
\hline Alachlor + atrazine & $8.23 \mathrm{~L}$ & 4940 & 7466 & - \\
\hline Mesotrione & $0.47 \mathrm{~L}$ & 4851 & 7727 & 8970 \\
\hline Thiencarbazone-methyl + isoxaflutole & $0.19 \mathrm{~L}$ & 5256 & 7318 & 9494 \\
\hline Acetochlor + atrazine & $8.23 \mathrm{~L}$ & 4915 & 7031 & 8899 \\
\hline S-Metolachlor & $1.59 \mathrm{~L}$ & 5143 & 7082 & 8806 \\
\hline Dimethenamid-P + atrazine & $4.12 \mathrm{~L}$ & 5294 & 8350 & 9611 \\
\hline Acetochlor (74.8\%) & $8.23 \mathrm{~L}$ & - & - & 8738 \\
\hline Rimsulfuron + mesotrione & $0.47 \mathrm{~L}$ & 4972 & 8295 & - \\
\hline Rimsulfuron + thifensulfuron-methyl & $0.09 \mathrm{~L}$ & 5168 & 7991 & 7934 \\
\hline$S$-Metolachlor + atrazine + mesotrione & $7.02 \mathrm{~L}$ & 5589 & 8556 & 9962 \\
\hline Dimethenamid-P & $1.22 \mathrm{~L}$ & 5275 & 7172 & 9447 \\
\hline Pendimethalin & $3.55 \mathrm{~L}$ & 5264 & 7881 & 8958 \\
\hline Saflufenacil & $0.19 \mathrm{~L}$ & 4524 & 8311 & 7477 \\
\hline Saflufenacil + dimethenamid-P & $1.12 \mathrm{~L}$ & 4906 & 7495 & 9377 \\
\hline Acetochlor $(33 \%)$ & $3.55 \mathrm{~L}$ & 5099 & 8310 & 8691 \\
\hline S-Metolachlor + mesotrione & $7.02 \mathrm{~L}$ & 5501 & 6160 & 8695 \\
\hline Pyroxasulfone & $210.16 \mathrm{G}$ & 5346 & 7548 & 9691 \\
\hline Untreated & - & 4506 & 6816 & 8218 \\
\hline $\operatorname{LSD}(0.05)$ & & 796 & 1800 & 1969 \\
\hline
\end{tabular}

${ }^{\mathrm{a}} \mathrm{L}=$ liters; $\mathrm{G}=$ grams.

Corn Injury. Early-season crop injury consisted of stunting and was never more than $3 \%$ with any herbicide treatment (including the $2 \mathrm{x}$ rate of acetochlor). Corn recovered from the slight early-season stunting and typically by harvest no differences in corn plant growth between the untreated check and any herbicide treatments were noted (data not shown). Although no appreciable crop injury was noted in these studies this is not always true. Instances of isoxaflutole phytotoxicity in corn have been documented [31,32] and attributed to several factors, including application timing [48], high use rate [49], and varied susceptibility of corn hybrids to isoxaflutole [50]. Environmental factors (cool and wet) and soil characteristics [49] can also lead to corn injury by isoxaflutole. Johnson et al. [32] reported that preplant incorporated herbicide applications resulted in greater injury than PRE applications and this was probably due to high amount of precipitation. Armel et al. [43] reported that acetochlor, atrazine, or mesotrione combinations caused 11 to $18 \%$ corn stunting when followed by $32 \mathrm{~mm}$ of rainfall but that the corn recovered quickly and by four weeks after treatment injury did not exceed $2 \%$.

Corn Yield. In 2013 at the Taylor location, atrazine, isoxaflutole, and pyroxasulfone alone and $S$-metolachlor plus either atrazine or mesotrione produced grain yields that were greater than the untreated check (Table 6). Despite not being significant, all herbicide treatments resulted in a numerical increase in grain yield over the untreated check. At the Ganado location, grain yields from the herbicide treatments were not significantly different from the untreated check; however, all yields from the herbicide treatments were numerically higher than the untreated check with the exception of $S$-metolachlor plus mesotrione which produced a $10 \%$ decrease in yield from the untreated check. No reason for this reduction can be determined.

In 2014, no significant differences between the untreated check and any herbicide treatments were noted although several herbicide treatments produced numerically higher yields than the untreated check (Table 5). Dimethenamid-P and pyroxasulfone alone, fluthiacet-methyl plus pyroxasulfone, thiencarbazone-methyl plus isoxaflutole, dimethenamid-P plus atrazine, $S$-metolachlor plus atrazine plus mesotrione, and saflufenacil plus dimethenamid-P produced grain yields that were 14 to $21 \%$ greater than the untreated check.

With glyphosate-resistant Amaranth spp. becoming more widespread throughout the state, the use of soil-applied herbicides can not only control resistant weed species in glyphosate-resistant corn production systems but also reduce 
the risk of new herbicide-resistant weed species occurring. In general, many treatments with two or three herbicides provided better weed control than one herbicide alone and the chance of corn injury appears to be minimal with any herbicide combinations under normal growing conditions. Our results indicate that in a year with little or no rainfall within 7 to $14 \mathrm{~d}$ after PRE herbicide application any combination of PRE herbicides may need to be followed by POST herbicides for control of escaped weeds.

\section{Competing Interests}

The authors declare that there are no competing interests regarding publication of this paper.

\section{References}

[1] G. Gullickson, "6 Points to remember about atrazine," 2012, http://www.agriculture.com/.

[2] M. S. Wiggins, M. A. McClure, R. M. Hayes, and L. E. Steckel, "Integrating cover crops and POST herbicides for glyphosateresistant palmer amaranth (Amaranthus palmeri) control in corn," Weed Technology, vol. 29, no. 3, pp. 412-418, 2015.

[3] Monsanto Company, Monsanto Biotechnology Trait Acres: Fiscal Years 1996-2009, 2009, http://www.monsanto.com/investors/ documents/2009/q4_biotech_acres.pdf.

[4] A. S. Culpepper, "Glyphosate-induced weed shifts," Weed Technology, vol. 20, no. 2, pp. 277-281, 2006.

[5] M. D. K. Owen, "Weed species shifts in glyphosate-resistant crops," Pest Management Science, vol. 64, no. 4, pp. 377-387, 2008.

[6] A. S. Culpepper and A. C. York, "Weed management in glyphosate tolerant cotton," Journal of Cotton Science, vol. 2, pp. 174-185, 1998.

[7] T. E. Klingaman and L. R. Oliver, "Palmer amaranth (Amaranthus palmeri) interference in soybeans (Glycine max)," Weed Science, vol. 42, no. 4, pp. 523-527, 1994.

[8] P. Sutton, C. Richards, L. Buren, and L. Glasgow, "Activity of mesotrione on resistant weeds in maize," Pest Management Science, vol. 58, no. 9, pp. 981-984, 2002.

[9] C. J. Swanton, R. H. Gulden, and K. Chandler, "A rationale for atrazine stewardship in corn," Weed Science, vol. 55, no. 1, pp. 75-81, 2007.

[10] J. D. Vyn, C. J. Swanton, S. E. Weaver, and P. H. Sikkema, "Control of Amaranthus tuberculatus var. rudis (common waterhemp) with pre and post-emergence herbicides in Zea mays L. (maize)," Crop Protection, vol. 25, no. 9, pp. 1051-1056, 2006.

[11] M. J. Walsh, K. Stratford, K. Stone, and S. B. Powles, "Synergistic effects of atrazine and mesotrione on susceptible and resistant wild radish (Raphanus raphanistrum) populations and the potential for overcoming resistance to triazine herbicides," Weed Technology, vol. 26, no. 2, pp. 341-347, 2012.

[12] J. D. Bollman, C. M. Boerboom, R. L. Becker, and V. A. Fritz, "Efficacy and tolerance to HPPD-inhibiting herbicides in sweet corn," Weed Technology, vol. 22, no. 4, pp. 666-674, 2008.

[13] D. O. Stephenson IV and J. A. Bond, "Evaluation of thiencarbazone-methyl- and isoxaflutole-based herbicide programs in corn," Weed Technology, vol. 26, no. 1, pp. 37-42, 2012.
[14] NRCS-National Resources Conservation Service, Plants Database: Amaranthus L., 2011, http://plants.usda.gov/java/profile? symbol=AMARA.

[15] W. J. Grichar, "Control of palmer amaranth (Amaranthus palmeri) in peanut (Arachis hypogaea) with postemergence herbicides," Weed Technology, vol. 11, no. 4, pp. 739-743, 1997.

[16] Weed Identification Guide, Southern Weed Science Society, Champaign, Ill, USA, 1990.

[17] Palmer amaranth-weed of TWO years-2014-2015, 2015, https://www.ag.ndsu.edu/weeds/weed-of-the-year-files/palmeramaranth-2.

[18] D. S. Correll and M. C. Johnston, Manual of the Vascular Plants of Texas, University of Texas at Dallas, Richardson, Tex, USA, 2nd edition, 1979.

[19] G. G. Light, M. Y. Mohammed, P. A. Dotray, J. M. Chandler, and R. J. Wright, "Glyphosate-resistant common waterhemp (Amaranthus rudis) confirmed in Texas," Weed Technology, vol. 25, no. 3, pp. 480-485, 2011.

[20] A. S. Franssen, D. Z. Skinner, K. Al-Khatib, M. J. Horak, and P. A. Kulakow, "Interspecific hybridization and gene flow of ALS resistance in Amaranthus species," Weed Science, vol. 49, no. 5, pp. 598-606, 2001.

[21] J. D. Sauer, "Recent migration and evolution of the dioecious amaranths," Evolution, vol. 11, no. 1, pp. 11-31, 1957.

[22] B. A. Sellers, R. J. Smeda, W. G. Johnson, J. A. Kendig, and M. R. Ellersieck, "Comparative growth of six Amaranthus species in Missouri," Weed Science, vol. 51, no. 3, pp. 329-333, 2003.

[23] J. A. Bond and L. R. Oliver, "Comparative growth of Palmer amaranth (Amaranthus palmeri) accessions," Weed Science, vol. 54, no. 1, pp. 121-126, 2006.

[24] M. J. Horak and T. M. Loughin, "Growth analysis of four Amaranthus species," Weed Science, vol. 48, no. 3, pp. 347-355, 2000.

[25] P. E. Keeley, C. H. Carter, and R. J. Thullen, "Influence of planting date on growth of Palmeramaranth (Amaranthus palmeri)," Weed Science, vol. 35, no. 2, pp. 199-204, 1987.

[26] A. S. Franssen, D. Z. Skinner, K. Al-Khatib, and M. J. Horak, "Pollen morphological differences in Amaranthus species and interspecific hybrids," Weed Science, vol. 49, no. 6, pp. 732-737, 2001.

[27] P. Guo and K. Al-Khatib, "Temperature effects on germination and growth of redroot pigweed (Amaranthus retroflexus), Palmer amaranth (A. palmeri), and common waterhemp (A. rudis)," Weed Science, vol. 51, no. 6, pp. 869-875, 2003.

[28] I. Heap, The International Survey of Herbicide Resistant Weeds, 2014, http://www.weedscience.com.

[29] D. O. Stephenson, J. A. Bond, R. L. Landry, and H. M. Edwards, "Weed management in corn with postemergence applications of tembotrione or thiencarbazone: tembotrione," Weed Technology, vol. 29, no. 3, pp. 350-358, 2015.

[30] SAS Institute Incorporated, SAS/STAT User's Guide: Statistics, Version 9.1, SAS Institute, Cary, NC, USA, 2007.

[31] S. Taylor-Lovell and L. M. Wax, "Weed control in field corn (Zea mays) with RPA 201772 combinations with atrazine and Smetolachlor," Weed Technology, vol. 15, no. 2, pp. 249-256, 2001.

[32] W. G. Johnson, G. S. Chahal, and D. L. Regehr, "Efficacy of various corn herbicides applied preplant incorporated and preemergence," Weed Technology, vol. 26, no. 2, pp. 220-229, 2012.

[33] Crop Protection Reference, Chemical \& Pharmaceutical Press, New York, NY, USA, 29th edition, 2014, http://www.greenbook .net. 
[34] G. A. Buchanan, D. S. Murray, and E. W. Hauser, "Weeds and their control in peanuts," in Peanut Science and Technology, $\mathrm{H}$. E. Pattee and C. T. Young, Eds., pp. 209-249, American Peanut Research Education Society, Yoakum, Tex, USA, 1982.

[35] R. W. Chamblee, L. Thompson Jr., and T. M. Bunn, "Management of broadleaf signalgrass (Brachiaria platyphylla) in peanuts (Arachis hypogaea)," Weed Science, vol. 30, pp. 30-40, 1982.

[36] J. W. Wilcut, G. R. Wehtje, and M. G. Patterson, "Economic assessment of weed control systems for peanuts (Arachis hypogaea)," Weed Science, vol. 35, pp. 433-437, 1987.

[37] J. W. Wilcut, A. C. York, and G. R. Wehtje, "The control and interaction of weeds in peanut (Arachis hypogaea)," Reviews of Weed Science, vol. 6, pp. 177-205, 1994.

[38] J. W. Wilcut, A. C. York, W. J. Grichar, and G. R. Wehtje, "The biology and management of weeds in peanut (Arachis hypogaea)," in Advances in Peanut Science, H. E. Pattee and H. T. Stalker, Eds., pp. 207-244, American Peanut Research Education Society, Stillwater, Okla, USA, 1995.

[39] M. A. Ross and D. J. Childs, Herbicide Mode-of-Action Summary, WS-23-W, Cooperative Extension Service, Purdue University, West Lafayette, Ind, USA, 1996, http://www.extension .purdue.edu/extmedia/ws/ws-23-w.html.

[40] W. J. Grichar, A. E. Colburn, and N. S. Kearney, "Herbicides for reduced tillage production in peanut (Arachis hypogaea) in the Southwest," Weed Technology, vol. 8, no. 2, pp. 212-216, 1994.

[41] G. L. Steele, P. J. Porpiglia, and J. M. Chandler, "Efficacy of KIH-485 on Texas panicum (Panicum texanum) and selected broadleaf weeds in corn," Weed Technology, vol. 19, no. 4, pp. 866-869, 2005.

[42] “KIH-485 herbicide," Technical Information, K-I Chemical U. S. A., White Plains, NY, USA, 2003.

[43] G. R. Armel, H. P. Wilson, R. J. Richardson, and T. E. Hines, "Mesotrione, acetochlor, and atrazine for weed management in corn (Zea mays)," Weed Technology, vol. 17, no. 2, pp. 284-290, 2003.

[44] G. R. Armel, H. P. Wilson, R. J. Richardson, and T. E. Hines, "Mesotrione combinations in no-till corn (Zea mays)," Weed Technology, vol. 17, no. 1, pp. 111-116, 2003.

[45] G. A. Ohmes, J. A. Kendig, R. L. Barham, and P. M. Ezell, "Efficacy of ZA1296 in corn," in Proceedings of the 53rd Annual Meeting of Southern Weed Science Society, p. 225, SWSS, Tulsa, Okla, USA, January 2000.

[46] J. R. Allen, W. G. Johnson, R. J. Smeda, and R. J. Kremer, "Alsresistant Helianthus annuus interference in Glycine max," Weed Science, vol. 48, no. 4, pp. 461-466, 2000.

[47] K. Al-Khatib, D. E. Peterson, and D. L. Regehr, "Control of imazethapyr-resistant common sunflower (Helianthus annuus) in soybean (Glycine max) and corn (Zea mays)," Weed Technology, vol. 14, no. 1, pp. 133-139, 2000.

[48] C. L. Sprague, J. J. Kells, and D. Penner, "Effect of application timing on corn tolerance and weed control with isoxaflutole," in Proceedings of the Weed Science Society of America (WSSA '97), Orlando, Fla, USA, 1997.

[49] P. W. Geier and P. W. Stahlman, "Efficacy of isoxaflutole alone and in combination in corn," in Proceedings of the North Central Weed Science Society Louisville (NCWSS '97), Louisville, KY, USA, 1997.

[50] C. L. Sprague and D. Penner, "Basis for different corn tolerance of four corn hybrids to isoxaflutole," in Proceedings of the North Central Weed Science Society Annual Meeting, p. 94, NCWSS, St. Paul, Minn, USA, 1998. 


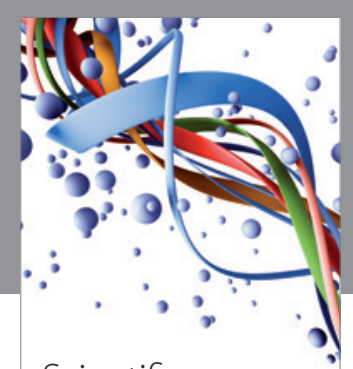

Scientifica
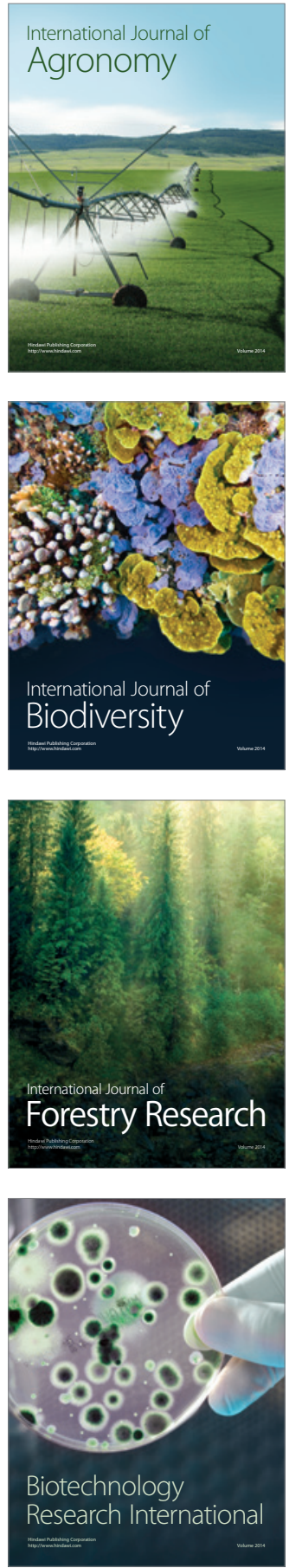
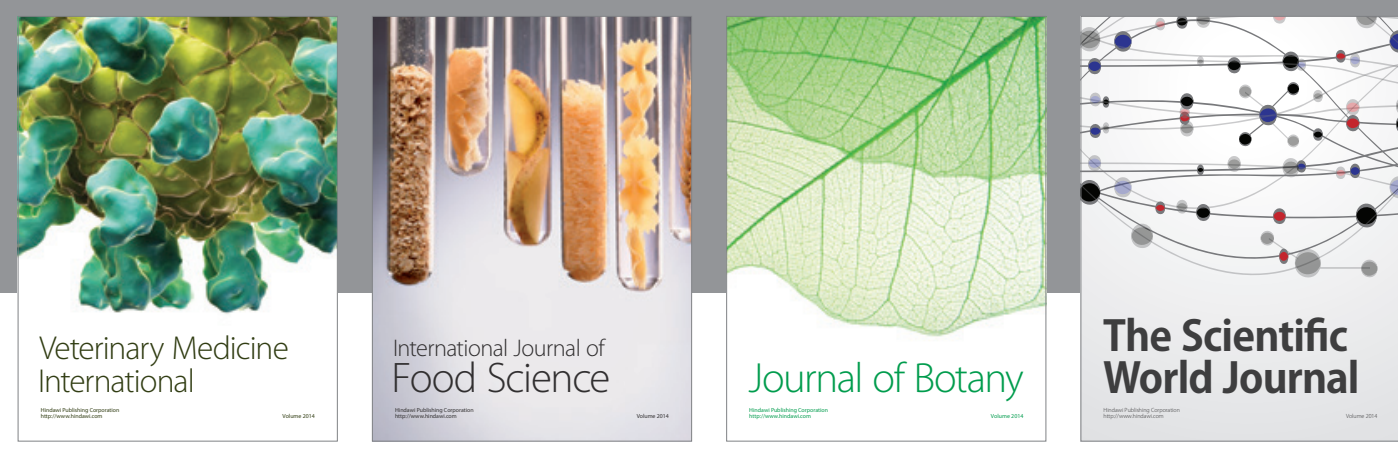

The Scientific

\section{World Journal}

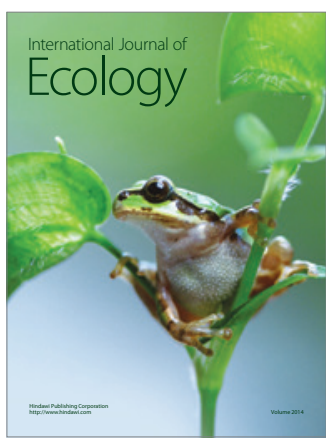

\section{Hindawi}

Submit your manuscripts at

http://www.hindawi.com
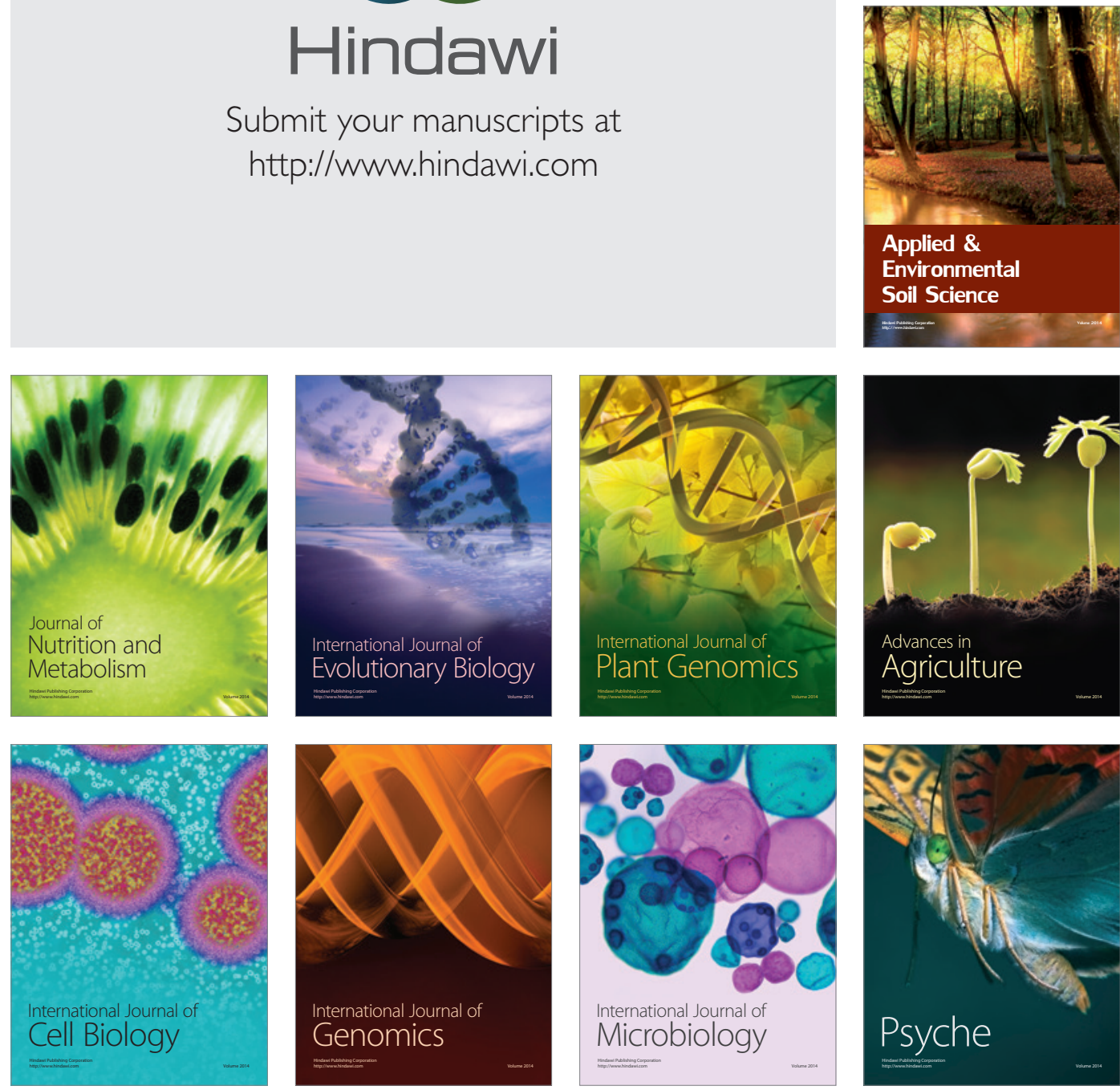
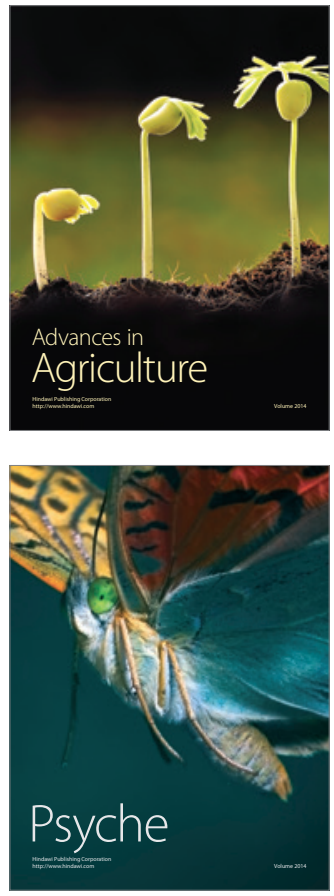